\title{
Estrategias para el control del estrés empresarial. Un estudio longitudinal en una empresa mediana.
}

\author{
Strategies for the control of business stress. A longitudinal study in a medium-sized \\ company.
}
Estrategias para o controle do stress corporativo: Um estudo longitudinal em uma empresa de médio porte.

Stratégies pour le contrôle du stress en entreprise. Une étude longitudinale dans une entreprise de taille moyenne.

José Vili Martínez González¹, Andrea García Valerio², Vili Aldebarán Martínez García

DOI: $10.19136 / h s . v 16 i 1.1388$

\begin{abstract}
ARTICULO ORIGINAL
Fecha de recibido: 9 de agosto de 2016 Fecha de aceptado: 29 de septiembre de 2016 Autor de correspondencia

José Vili Martínez González. Dirección postal: Ajedrez Consultores, Adolfo López Mateos No. 46 Col. Santa Clara Coatitla, Estado de México. C.P. 55540. Correo electrónico: ajedrezconsultores@hotmail.com
\end{abstract}

\section{Resumen}

Objetivo: Identificar si las estrategias propuestas en la unidad de estudios seleccionada coadyuvan a la disminución del estrés.

Materiales y Métodos: Investigación descriptiva, de diseño no experimental, longitudinal, de evolución de grupo. La muestra no probabilística considera a un total de 20 ejecutivos y 40 operativos, empleando un instrumento que cuenta con validez y confiabilidad de coeficiente de alfa Cronbach de 0.7933 , compuesto por tres dimensiones y 78 reactivos.

Resultados: Se identificaron disminuciones importantes en las variables de ambiente físico y organizacionales, moderadas en síntomas y bajas en extra-organizacionales.

Conclusiones: Un programa bien estructurado, medido y dirigido permite disminuir los efectos negativos del estrés en las organizaciones y por añadidura, beneficia a los sistemas de salud al disminuir los costos relacionados con la atención de los males relacionados con el estrés.

Palabras claves: Estrés empresarial, ambiente laboral, mediana empresa.

1.Doctor en Administración. Ajedrez Consultores.

2.Maestra en Seguridad e Higiene Ocupacional. Ajedrez Consultores.

3.Participante de Formación Temprana de Científicos Proyecto Savia, del CONACyT y SIIES y parte del equipo de Ajedrez Consultores. 


\section{Abstract}

Objective: To identify if the strategies proposed in the unit of selected studies contribute to the reduction of stress.

Materials and Methods: Descriptive research, non-experimental design, longitudinal, evolution of the group. The non-probabilistic sample considers a total of 20 executives and 40 operational, using an instrument that has validity and reliability of Cronbach's alpha coefficient of 0.7933 , composed of three dimensions and 78 reagents.

Results: We identified significant declines in the variables of physical environment and organizational, moderate symptoms and low in extraorganizational.

Conclusions: A well-structured program, measured and directed allows to decrease the negative effects of stress in the organizations and in addition, benefits to health systems by lowering the costs related to the care of the evils related to the stress.

Keywords: Business Stress, working environment, medium-sized company.

\section{Resumo}

Objetivo: Identificar se as estratégias propostas na unidade de estudos selecionada podem contribuir para a redução do stress.

Materiais e métodos: Pesquisa descritiva, não experimental, longitudinal e de evolução de grupo. A amostra não aleatória considera um total de 20 executivos e 40 funcionários operacionais, usando um instrumento com validade e confiabilidade do coeficiente de alfa Cronbach de 0,7933, composto por três dimensões e 78 reagentes.

Resultados: Foram identificadas reduções significativas nas variáveis ambiente físico e organizacionais, moderadas nos síntomas e baixas nas extraorganizacionais.

Conclusões: Um programa bem estruturado, medido e gerenciado pode reduzir os efeitos negativos do stress nas organizações, assim como beneficiar os sistemas de saúde devido à redução dos custos relacionados com o atendimento e tratamento de doenças relacionadas com o stress.

Palavras-chave: Stress nas empresas, ambiente de trabalho, empresas de porte médio.

\section{Résumé}

Objectif: Identifier si les stratégies proposées dans l'unité d'études sélectionnée contribuent à la réduction du stress.

Matériels et méthodes: Étude descriptive, non expérimentale, longitudinale, d'évolution de groupe. L'échantillon non aléatoire inclut un total de 20 cadres et 40 employés ou ouvriers. L'instrument utilisé est composé de trois dimensions et 78 réactifs. Sa validité et sa fiabilité correspondent à un coefficient alpha de Cronbach de 0,7933 .

Résultats: Des diminutions importantes ont été observées pour les variables environnement physique et organisationnelles, modérées pour la variable symptômes, et faibles pour la variable extra-organisationnelles.

Conclusions: Un programme bien structuré, mesurée et ciblée permet de réduire les effets négatifs du stress dans les organisations et, de plus, présente l'avantage pour les systèmes de santé de réduire les coûts liés à la prise en charge des problèmes liées au stress.

Mots-clés: stress en entreprise, environnement laboral, entreprises moyennes. 


\section{Introduccion}

En la actualidad, existe un consenso en torno a la importancia de controlar y disminuir el estrés en las organizaciones y un número importante de empresas llevan a cabo acciones específicas para buscar controlarlo y disminuirlo; sin embargo, muchas de estas iniciativas no cuentan con indicadores que permitan identificar su eficacia y por ende, terminan evaluando sus acciones tan solo por la "sensación" de que las cosas han mejorado. Por esto, resulta fundamental contar con una herramienta que sea de utilidad para medir si las acciones emprendidas han dado resultado. "El estrés laboral está siendo cada vez más visualizado como un problema de salud." 1

Un estudio realizado por la Organización Internacional del Trabajo $(\mathrm{OIT})^{2}$ muestra que la salud mental en el trabajo se encuentra amenazada, mencionando entre otros efectos fácilmente identificables, que los empleados sufren agotamiento, ansiedad, un bajo estado de ánimo y estrés; dichos factores pueden coadyuvar en descuidos y fallas en el trabajo. Por ello, es factible intuir que los empresarios obtendrán una menor productividad, reducción de beneficios, y contrario a sus deseos, se pueden ver retribuidos con elevados índices de rotación del personal y fallas relacionadas con el cansancio y el estrés. Estas afeccciones se magnifican cuando hablamos a nivel de costos de gobierno, ya que esta epidemia se traduce en un aumento de los costos en la asistencia sanitaria y una disminución de la renta nacional.

Sin embargo, conceptualizar el estrés es una cuestión muy compleja y ha sido sujeta a múltiples análisis entre diferentes expertos de diversas áreas, ya que es una alteración de todos los sistemas adaptativos del organismo y entran en acción diversos ejes hormonales que se alteran.

Desde un punto de vista fisiológico, ¿estrés? es cualquier situación que pone al cuerpo en estado de alerta. ${ }^{3}$

El Hospital General de México menciona que "Se trata de estados de malestar subjetivo, acompañados de alteraciones emocionales que, por lo general, interfieren con la actividad social y que aparecen en el periodo de adaptación a un cambio biográfico significativo o de un acontecimiento vital estresante." 4

Para la Organización Internacional del Trabajo (OIT), "es la respuesta física y emocional dañina causada por un desequilibrio entre las exigencias percibidas y los recursos y capacidades percibidos de un individuo para hacer frente a esas exigencias." 5

El principal factor de riesgo es la presencia de un agente estresante. El cuadro suele comenzar un mes posterior a estar en contacto con el evento estresante; es muy variable y puede cursar con: depresión, ansiedad, preocupación, sentimiento de incapacidad para afrontar problemas, deterioro de la rutina diaria, incapacidad para afrontar el futuro, trastornos disociales. El trastorno adaptativo se manifiesta frecuentemente a través de una disminución de rendimiento en el trabajo o en la escuela. Estos trastornos están asociados con aumento de riesgo de suicidio, o pueden complicar el curso de una enfermedad del individuo. El tratamiento está basado en la interrupción del factor estresante, acompañado de psicoterapia.

No sólo se invierte una gran cantidad de tiempo en el trabajo; mucha gente encuentra que es una parte sustancial de su satisfacción y de su identidad. Consecuentemente, su vida laboral y no laboral se entrelazan resultando que son interdependientes, a grado tal, que la casa se convierte en una extensión de su organización; en este sentido es factible mencionar que el estrés ocupacional prevalece en casi todas las organizaciones laborales de trabajo y está provocando alteraciones en la salud.

El trabajo industrial ha traído consigo una problemática compleja que alude, no sólo a la salud física, sino también a la mental cuya preocupación en el medio laboral, ha llevado a los estudiosos a indagar concretamente los efectos nocivos $\mathrm{y}$ adversos que emergen del propio proceso productivo.

El resultado es que los procesos industriales y el cambio tecnológico, están generando importantes cambios en el patrón de desgaste de los trabajadores industriales y no industriales. $^{6}$ Se identifican, en consecuencia, problemas emergentes relacionados con la salud mental y/o emocional, relacionados con condiciones de trabajo peligrosas, pero también con bajo esfuerzo, monotonía y aislamiento, cuya expresión está conformando una patología psicosomática y nerviosa que, en términos generales, se asume como estrés.

La exposición a condiciones estresantes en el trabajo puede tener una influencia directa en la salud y seguridad de los trabajadores. Las inadecuadas condiciones de trabajo, los conflictos constantes con supervisores o el hostigamiento intencional de los compañeros, pueden llegar originar neurosis, angustia y en casos extremos, suicidio.

Las organizaciones necesitan identificar los trabajos que llevan a una fatiga laboral prematura y las personas que manifiestan algunos de los síntomas tempranos de este problema. A veces será posible modificar las partes de un trabajo que favorece la fatiga laboral. En otros casos, la empresa puede ayudar a los empleados a sortear mejor las situaciones laborales de estrés, considerando que en primera instancia se pueden realizar cambios de ingeniería, 
procesos administrativos y finalmente dotar al personal con equipo de protección personal que ayuden a mitigar o disminuir los factores estresantes.

El National Institute of Mental Health señala que las principales causas del estrés de los empleados se dividen de manera uniforme entre los factores organizacionales y el ambiente ajeno al trabajo. Esas causas de origen doble, muestran que los empleados pueden responder a estos factores estresantes en forma positiva (que los estimula), o con un estrés negativo (que disminuye sus esfuerzos.) De modo que, pueden originarse consecuencias constructivas o destructivas tanto para la organización como para el individuo.?

Es posible conceptualizar a una organización saludable como aquella que presenta bajos niveles de enfermedad, lesiones y discapacidad en su fuerza de trabajo y también por la competitividad en el mercado. Las investigaciones del Instituto Nacional para la Seguridad y Salud Ocupacional (NIOSH) han identificado las características organizacionales asociadas, tanto en el trabajo saludable bajo en estrés, como los altos niveles de productividad.

Los ejemplos de estas características incluyen las siguientes:

1.-Reconocimiento a los trabajadores por el buen desempeño laboral;

2.-Oportunidades para el desarrollo de una carrera;

3.-Una cultura organizacional que valora el trabajo individual;

4.-Acciones de la gerencia que son congruentes con los valores organizacionales. ${ }^{8}$

Una opción viable en las organizaciones es brindar capacitación para el manejo del estrés y un programa de apoyo al trabajador para enfrentarse con situaciones difíciles en el trabajo.

Existen cuatro razones por las cuales deben saber cómo afrontar el estrés:

1.- Para mejorar la salud del empleado;

2.- La productividad;

3.- La creatividad;

4.- El rendimiento sobre la inversión.

Un gerente eficaz nunca ignora un problema de rotación de personal o de ausentismo, una baja en la calidad o cantidad de la producción o cualquier otro síntoma de que las metas de la organización no están siendo alcanzadas en la forma más eficaz y oportuna, ve estos acontecimientos como síntomas y va más allá de ellos para identificar y corregir las causas subyacentes. Sin embargo, probablemente reconocerá como causas, únicamente aquellas que se le enseñó a reconocer, y se da el caso de que el estrés no está en la lista de posibles sospechosos, por lo que no se le diagnostica ni prescribe.

Por lo tanto, el primer paso en el manejo efectivo del estrés es el reconocimiento o la concientización de que el estrés puede ser una causa subyacente en los problemas de trabajo individuales y grupales, y en segundo lugar los individuos que están experimentando estrés, deben reconocerlo. ${ }^{9}$

Ramos $^{10}$ menciona que para disminuir el estrés se debe llevar a cabo un programa que consiste en:

1.- Cambio organizacional. Otras empresas tratan de reducir el estrés del trabajo llevando consultores para recomendar formas de mejorar las condiciones de trabajo. Este enfoque es la forma más directa de reducir el estrés en el trabajo. Involucra la identificación de los aspectos estresores del trabajo (carga de trabajo excesiva, expectativas conflictivas);

2.- El diseño de estrategias para reducir o eliminar los estresores identificados. La ventaja de este enfoque es que conduce directamente a la raíz de las causas del estrés en el trabajo. Sin embargo, algunas veces los administradores están incómodos con este enfoque porque puede involucrar cambios en las rutinas de trabajo o calendarios de producción, o cambios en la estructura.

\section{El Center for Disease Control and Prevention ${ }^{11}$ propone:}

1.- Asegure que el volumen de trabajo coordine con las habilidades y los recursos de los trabajadores;

2.- Diseñe los trabajos para proveer el significado, el estímulo, y las oportunidades para que los trabajadores usen sus habilidades;

3.- Defina claramente los papeles y responsabilidades de los trabajadores;

3.- Dé oportunidades a los trabajadores de participar en las decisiones y acciones afectando sus trabajos;

4.- Mejore las comunicaciones -reduzca la incertidumbre sobre el desarrollo de carrera y las posibilidades de trabajo en el futuro;

5.- Provea oportunidades para la interacción social entre los trabajadores;

6.- Establezca los calendarios de trabajo que están compatibles con las demandas y responsabilidades fuera del trabajo.

fronteriza. 


\section{Materiales y métodos}

La presente investigación es de tipo descriptiva, de diseño no experimental, longitudinal, de evolución de grupo, en la cual se busca identificar si las estrategias empleadas por la unidad de estudio resultaron útiles para disminuir los factores identificados como estresantes.

La muestra es no probabilística, considera a un total de 20 ejecutivos (seleccionados considerando a los directivos con menor posibilidad de rotación) y 40 operativos (los cuales fueron seleccionados considerando a los que contaban con base).

La empresa se clasificó como mediana en el sector industrial, al contar con menos de 250 trabajadores, considerando el criterio de Consejo Nacional de Ciencia y Tecnología (CONACyT) $)^{12}$

El instrumento usado (Aldebarán-Test) ${ }^{13}$ cuenta con validez y confiabilidad de coeficiente de alfa Cronbach de 0.7933 , compuesto por tres dimensiones y 78 reactivos.

Las hipótesis consideradas fueron:

$\mathrm{H}_{1}$ : Las estrategias implementadas por la organización no disminuyeron el estrés en los participantes de la muestra seleccionada.

$\mathrm{H} 1_{1}$ : Las estrategias implementadas por la organización si disminuyeron el estrés en los participantes de la muestra seleccionada.

En lo relativo a la aplicación del instrumento, esto se realizó en 12 sesiones considerando grupos integrados en promedio por 5 participantes, aplicando la primera encuesta en mayo del 2015 y la segunda en mayo del 2016

En lo relativo a las estrategias implementadas por la organización para disminuir el estrés es posible resumirlas en:

1. Se organizó y limpió toda la planta en general empleando la herramienta 5 "S" +1 ;

2. Aplicación de KAN-BAN que es una técnica japonesa que consiste en organizar la planta, disminuir demoras por malos acomodos, mejora en procesos y mejora continua;

3. Se sustituyeron y organizaron los racks para materia prima y producto final;

4. Dentro de la logística, las camionetas de entrega se cargan por la tarde, de esta manera al llegar en la mañana pueden salir a repartir inmediatamente, lo que evita retrasos en entregas y permite que los choferes lleguen a tiempo para cargar y salir a la hora destinada, evitando con ello fricciones y estrés al interior de la organización;

5. Se entregó equipo de protección personal y se dio la capacitación sobre su correcto uso;

6. Modificación de equipo obsoleto por equipo nuevo y reciente (camionetas de carga y equipo de cómputo);

7. Capacitación interna (primeros auxilios, calidad, actitud proactiva, estrés empresarial, son algunos de los cursos que se les ha ofrecido);

8. Monitoreo de productividad a través de objetivos mensuales que son anunciados en un pizarrón a la vista de todos;

9. Instalación y puesta en marcha de un comedor;

10. Instalación de un área para ejercicio tanto para actividades cardiovasculares como una zona de pesas;

\section{Incremento salarial desde $5 \%$ hasta $20 \%$;}

12. Se cuenta con un consultor externo que apoya a lo largo de todo el proceso de cambio de la organización.

Se consideraron 5 variables de análisis: Síntomas, Ambiente Físico (AF), Organizacionales (O), Extra-organizacionales (E), y Estrés.

La cuales fueron definidas operacionalmente como:

1.- Síntomas: En este rubro se consideran los síntomas que se han vinculado con el estrés.

2.- Ambiente Físico: Son los que "se encuentran en el ambiente de trabajo, están directamente relacionados con los agentes contaminantes" comprendiendo a los contaminantes como todo factor que está relacionado con el estrés.

3.- Organizacionales: Son "los conflictos intra-grupales que pueden perturbar la efectividad de los grupos y dar como resultado un estrés a largo plazo"

4.- Extra-organizacionales: Son "las acciones, situaciones o los sucesos ajenos a la organización, que podrían resultar estresantes para la gente".

5.- El estrés: Se considera para este estudio como los indicadores que el instrumento seleccionado identifica, relativo a las reacciones fisiológicas y psicológicas que los participantes identifiquen que se hacen sobre ellos. 


\section{Resultados}

Los La estrategia implementada por la empresa logró excelentes resultados en dos de las variables medidas ya que disminuyo en $84.90 \%$ lo relacionado con los factores organizacionales y $62.50 \%$ de los factores del ambiente físico.

Se obtuvieron resultados moderados en lo relativo a los síntomas relacionados con el estrés ya que solo se logró una disminución de 55.31\%.

Bajos resultados en lo concerniente a los extraorganizacionales con una disminución de $26.31 \%$, si bien, los resultados en el área extra-organizacional fueron bajos, esta condición se explica en gran medida debido a que esta variable está compuesta por factores que están fuera del control de la unidad de estudio seleccionada.

Lo anterior se presenta con mayor detalle en la tabla 1 y es factible tener una mejor visión de los mismos en la figura 1.

\section{Discusión}

Tabla 1: Disminución de los estresores que se presentó en los trabajadores después de la implementación del programa.

\begin{tabular}{|l|l|l|}
\hline Variables & $\begin{array}{l}\text { Presencia identificada en la } \\
\text { muestra obtenida en 2016 }\end{array}$ & $\begin{array}{l}\text { Disminución en la muestra } \\
\text { seleccionada en relación con la } \\
\text { muestra obtenida en 2015 }\end{array}$ \\
\hline Síntomas & $44.68 \%$ & $55.31 \%$ \\
\hline Ambiente fisico & $37.50 \%$ & $62.50 \%$ \\
\hline Organizacionales & $15.09 \%$ & $84.90 \%$ \\
\hline Extra-organizacionales & $73.68 \%$ & $26.31 \%$ \\
\hline
\end{tabular}

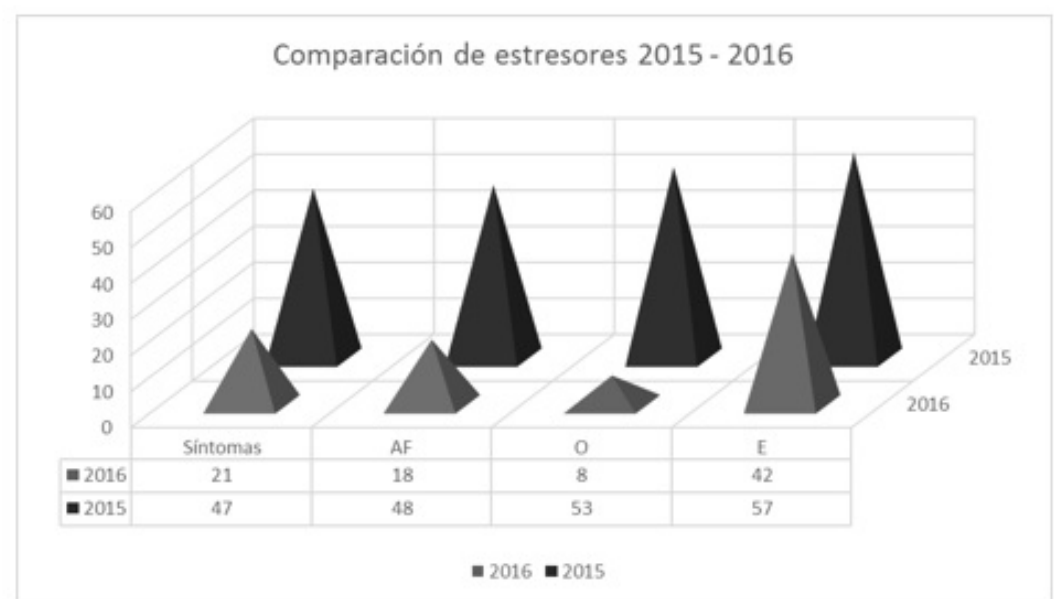

Figura 1: Comparación de tipo de estresores que se presentaron en el 2015 y 2016. 


\section{Conclusiones}

Se acepta la hipótesis:

$\mathrm{H}_{1}$ : Las estrategias implementadas por la organización sí disminuyeron el estrés en los participantes de la muestra seleccionada.

$\mathrm{Si}$ bien los resultados obtenidos permiten visualizar fácilmente los beneficios de aplicar este tipo de programas bien estructurados es importante remarcar:

a) Dar continuidad a las estrategias emprendidas;

b) Continuar en la búsqueda de áreas de oportunidad;

c) Realizar mediciones constantes para conocer los avances o la necesidad de realizar ajustes al programa;

d) Compartir sus experiencias con otras empresas y realizar benchmarking para mejorar los resultados alcanzados;

e) Identificar una herramienta que permita cuantificar los beneficios económicos logrados a partir del programa implementado.

Con base en los datos obtenidos, es factible sugerir que un programa bien estructurado, medido y dirigido permite disminuir los efectos negativos del estrés en las organizaciones y por añadidura, beneficia a los sistemas de salud, al disminuir los costos relacionados con la atención de los males relacionados con el estrés.

Por otra parte, podemos ver que tanto la Organización Mundial de la Salud (OMS) como la OIT han emitido recomendaciones para la prevención de problemas mentales, debidos a los factores psicosociales generados en el trabajo, promoviendo una política de trabajo adecuada, integral y basada en principios éticos, para generar lugares de trabajos saludables

Se sugiere para futuras investigaciones, realizar estudios similares en otras organizaciones, lo que permitirá incrementar el marco teórico, así como realizar un estudio de seguimiento con en la unidad de investigación para identificar y comparar el comportamiento encontrado con las mediciones ya obtenidas.

\section{Referencias}

${ }^{1}$ Ramírez, J. Mujer, Trabajo y Estrés. Rev Latinam Salud Trab. 2001; 1 (2): 58-74.

${ }^{2}$ S.O.S. estrés en el trabajo: aumentan los costes del estrés en el trabajo y la incidencia de la depresión es cada vez mayor. La Revista de la OIT [Internet]. 2000 [Citado el 31 de julio de 2016]; 37:4-5.Disponible en: http://www.ilo.org/ wcmsp5/groups/public/---dgreports/---dcomm/documents/ publication/dwcms_080699.pdf

${ }^{3}$ Cuatrecasas, G. Estrés y dolor crónico: una perspectiva endocrinológica. Reumatología Clínica [Internet]. 2009 [Citado el 10 de agosto del 2016]; 5:12-14. Disponible en:http://www.reumatologiaclinica.org/es/estres-dolorcronico-una-perspectiva/articulo/S1699258X09001442/. DOI:10.1016/j.reuma.2009.04.001

${ }^{4}$ [Citado el 12 de enero de 2016]. Disponible en: www.hgm. salud.gob.mx/descargas/pdf/.../1trastornos_adaptativos.pdf

${ }^{5}$ Estrés en el trabajo: Un reto colectivo. OIT [Internet]. 2016 [Citado el 5 de agosto de 2016]. Disponible en: http://www. ilo.org/wcmsp5/groups/public/---ed_protect/---protrav/--safework/documents/publication/wcms_473270.pdf

${ }^{6}$ Ramírez, J. Mujer, Trabajo y Estrés. Rev Latinam Salud Trab. 2001; 1 (2): 58-74.

${ }^{7}$ Keith, D. Comportamiento Humano en el Trabajo. $8^{\mathrm{a}}$. ed. México: Mc. Graw Hill, 1993.

${ }^{8}$ Ramos, J. Estrés...en el Trabajo. Traducción. Rev Latinam Salud Trab. México. 2001; 1 (2): 88.

9Ivancevich, J. Estrés en el Trabajo. México: Trillas; 1989.

${ }^{10}$ Ramos, J. Estrés...en el Trabajo. Rev Latinam Salud Trab. 2001; 1(2): 86-90.

${ }^{11} \mathrm{CDC}$ - NIOSH Strategies for preventing job stress. Cdc.gov [Internet]. 2016 [Citado el 31 de julio de 2016]. Disponible: http://www.cdc.gov/niosh/updates/stresrel. html

${ }^{12}$ [Citado el 9 de agosto de 2016]. Disponible en: http:// www.cotacyt.gob.mx/descargables/Clasificacion $\% 20$ del $\% 20$ tama $\%$ C3\%B10\%20de $\% 201$ as $\% 20$ empresas.pdf

${ }^{13}$ García, A. Discriminación de factores de estrés laboral entre ejecutivos y personal operativo en una micro empresa del Valle de México (Maestría). Secretaría del Trabajo y Previsión Social. 2003.

${ }^{14}$ López, H. Enrique. Stress y Ambiente de Trabajo. Higiene y Seguridad. México. 1995:3-17.

${ }^{15}$ Ivancevich, J. Estrés en el Trabajo. México: Trillas; 1989.

${ }^{16}$ Ivancevich, J. Estrés en el Trabajo. México: Trillas; 1989.

${ }^{17}$ Mercado, P; Salgado, R. Estrés laboral en ejecutivos de medianas y grandes empresas mexicanas [Internet]. 2008 
[Citado el 10 de agosto de 2016]. Disponible en: http:// factorespsicosociales.com/segundoforo/trabajos_libres/ MERCADO-SALGADO.pdf

${ }^{18}$ Guía sobre el manejo y prevención del estrés laboral. Secretaria de Salud [Internet]. 2010 [Citado el 10 de agosto de 2016]. Disponible en: http://www.promocion. salud.gob.mx/dgps/descargas1/programas/Guia_Manejo_ Estres_30062010.pdf

${ }^{19}$ Mitchell, C. Estrés laboral es una carga para los individuos, los trabajadores y las sociedades. PanAmerican Health Organization / World Health Organization [Internet]. 2016. Disponible en: http://www.paho.org/hq/index. php?option $=$ com_content $\&$ view $=$ article $\&$ id $=11973 \% 3 \mathrm{Aw}$ orkplace-stress-takes-a-toll-on-individuals-employers-andsocieties\&Itemid=135\&lang=es 